DOI 10.35381/cm.v7i1.556

\title{
Propuesta de configuración de un servidor de impresiones
}

\section{Proposal to configure a print server}

\author{
Luis Orlando Albarracín-Zambrano \\ uq.luisalbarracin@uniandes.edu.ec \\ Universidad Regional Autónoma de los Andes, Quevedo \\ Ecuador \\ https://orcid.org/0000-0002-3164-5229 \\ Juan Carlos Lozada-Calle \\ juancarloslozada99@gmail.com \\ Universidad Regional Autónoma de los Andes, Quevedo \\ Ecuador \\ https://orcid.org/0000-0003-3124-0421 \\ Jennifer Pamela Martínez-Matute \\ pamemm-1992@hotmail.com \\ Universidad Regional Autónoma de los Andes, Quevedo \\ Ecuador \\ https://orcid.org/0000-0001-7241-0605
}

Recibido: 01 de agosto de 2021

Aprobado: 15 de octubre de 2021 


\title{
RESUMEN
}

La investigación abordó una problemática actual de reactivación económica y reingeniería de servicios que brindan las pequeñas empresas como los centros de cómputo, partiendo desde ¿Cómo mejorar la gestión de impresiones en la empresa "Centro de Computo Lumar System's" del Cantón La Maná - Cotopaxi?, donde se plantea la configuración de un servidor de impresión para la gestión de impresiones en la empresa, optimizando los recursos compartido de impresoras, para reducir gastos en papel, manejando la cola de impresiones, llevar un control de solicitudes de impresiones de cada una de las estaciones de trabajo, llevar un conteo de impresiones, el principal aporte que hace este trabajo es que se presenta un modelo de configuración de red para el control de impresiones que puede ser aplicada en cualquier Pyme, constituyendo una solución informática acorde a los avances de las nuevas tecnologías.

Descriptores: Empresa; impresión; centro de análisis de información. (Palabras del Tesauro UNESCO).

\begin{abstract}
The research addressed a current problem of economic reactivation and reengineering of services provided by small companies such as computer centers, starting from How to improve the management of impressions in the company "Centro de Computo Lumar System's" of Cantón La Maná - Cotopaxi? , where the configuration of a print server for print management in the company is proposed, optimizing the shared resources of printers, to reduce paper costs, managing the print queue, keeping track of print requests for each of workstations, keep a count of impressions, the main contribution that this work makes is that it presents a network configuration model for the control of impressions that can be applied in any SME, constituting a computer solution according to the advances of the new technologies.
\end{abstract}

Descriptors: Company; Print; information analysis center. (Words from the UNESCO Thesaurus). 


\section{INTRODUCCIÓN}

Desde la creación de las PYMES a finales de los ochenta, se ha venido buscando la especialización de las pequeñas y medianas empresas, prestando principal atención a aspectos específicos de gestión, y más concretamente aquellos relacionados con la tecnología e innovación y actualmente la anunciada vuelta a una normalidad permite pensar en una reactivación económica que por causas de la pandemia ha dejado muchos negocios en una inestabilidad económica. La automatización de los procesos dentro de las PYMES, ha tomado un papel preponderante dentro de la gestión, la clave no es tanto la comprensión de los fenómenos tecnológicos, como las consecuencias del impacto en la organización, en el uso de las técnicas de gestión, y la dirección, sino más bien el uso adecuado de la información que genera cada uno de los procesos, o se deberá hablar de un mejor conocimiento del uso de la tecnología y las posibilidades que brindan, debiendo realizar un análisis dentro de la empresa sobre la utilización de los ordenadores, comunicaciones, recursos compartidos, entre otras.

En la actualidad el panorama empresarial dentro del Ecuador no difiere de lo que está sucediendo fuera de él, pues el desarrollo tecnológico y la creación de PYMES, es una de las fuentes económicas en mayor auge, y la implementación de la tecnología dentro de ellas, así como, el manejo de la información, el uso de ordenadores, la comunicación de datos entre ordenadores y el uso correcto de los recurso compartidos(impresoras), para reducir gastos y mejor su gestión administrativa, basada en una correcta y adecuada aplicación y utilización de la tecnología. Actualmente brindan un servicio con ciertos problemas en proceso de impresión, cometiendo errores como, múltiples ordenes de impresión ocasionando congestión en la cola de impresiones, impresiones de información no útil, o "impresiones basura" representando para la empresa inconvenientes en la gestión de administración, en el proceso de cobro de sus servicios y en la administración de los recursos que brinda la empresa, así como pérdidas económicas y malestar en los clientes. 
Redes de comunicación de datos: Es una combinación de hardware, software y medios de transmisión con el objetivo de conectar dos o más nodos permitiendo el intercambio de información entre ellos. Como nodo se considera cualquier elemento conectado a la red (ordenador, periféricos, etc.), distinguiendo los nodos servidores (sistemas encargados de procesar y controlar a nivel lógico el intercambio de información en la red, así como su almacenamiento) de los nodos clientes (sistemas que hacen uso de los recursos de la red).

Servidor de impresión: El print server es un servicio que centraliza las impresiones de una red, recibiendo las peticiones enviadas desde los clientes que cuentan con acceso a la red y al servicio, esta petición la reenvía a la impresora. (Arias, 2019).

Los servidores de impresión permiten al usuario brindar el servicio de impresión a todos los nodos de la red soportando en esta varias interfases sean estas paralelo, serie o mixtas, el servidor de impresión ademas de compartir la impresora gestiona los trabajos de impresión enviados desde cualquier nodo de la red utilizando los protocolos que soporte ademas de utilizar la impresora apropiada para cada print job (trabajo de impresión).

Francisco Moliner en el libro Informatica para grupos indica que inicialmente los servidores de impresión unicamente soportaban un protocolo conectado generalmente en paralelo siendo estos dispositivos externo. Al respecto, Moliner (2005), afirma "De manera general los servidores de impresión no poseen una gran cantidad de memoria, en lugar de almacenar los trabajos de impresión ellos simplemente guardan la direccion de host y el protocolo involucrado en la cola". De esta forma se ganando con esto velocidad de impresión ademas de permitir a los servidores gestionar y direccionar los trabajos de impresión, de esta manera esperan hasta que la impresora necesaria este disponible enviando a ella en ese momento el trabajo colocado en la cola. En ese sentido, los servidores de impresión permiten impresoras para ser conectado a una red y se distribuyen entre varios usuarios. Hay también muchos beneficios adicionales, el ahorro y características que no todos pueden ser considerados de inmediato: 
Ahorro de espacio: Ya no es necesario comprar una impresora dedicada para cada usuario, por lo tanto, usted puede ahorrar el espacio, la electricidad y los costes de mantenimiento.

Instalación rápida: Agregar una impresora a la red en cuestión de minutos. No hay necesidad de cerrar su red.

La flexibilidad, evitando colas o retrasos: Redes más de una o todas las impresoras pueden ofrecer una gran flexibilidad:

1. Todos los usuarios podrían tener la opción de imprimir en color o de inyección de tinta de impresora láser.

2. Si una impresora está en uso con un trabajo de impresión grandes, entonces el usuario puede evitar la cola con cualquier otro de la impresora.

3. Impresora de tinta o tóner y alguien se olvidó de recambios? O simplemente no tienen tiempo o no saben qué hacer para cambiarlo.

4. Impresora defectuoso y está esperando a que alguien cheque / reparación / sustitución

\section{Topologías de las redes de comunicación}

Lo primero que caracteriza una red local es la manera en que se conectan las estaciones; es decir, la forma que adopta el medio compartido entre las mismas. Básicamente existen tres topologías posibles: topología en estrella, en bus, en anillo. Al seleccionar una topología se debe garantizar la operación eficiente y continua de la red, tomando en cuenta futuras expansiones. La topología de red afecta directamente al tráfico y el enrutamiento de datos que circulan dentro de ella (Mejías, 2021).

Topología de estrella. - A diferencia de la topología en bus, que tenía un solo canal de comunicaciones para todos los dispositivos, en esta, cada dispositivo de red tiene su propio canal. De ese modo, no se generan colapsos ni saturación (Sánchez y Bolaño, 2021).

El sistema está compuesto por una unidad de control, alrededor de la cual se distribuyen el resto de unidades de control. Si falla la unidad central, todas las 
transmisiones se interrumpirán. En este tipo de conexión es necesarito una gran cantidad de cableado (Domínguez, 2018).

Topología de bus. - Todos los nodos se conectan a la misma barra o bus a través de un transceptor, el cuales controla la comunicación. Toda la información se envía y se recibe a través del bus y los nodos la rechazan o la reciben dependiendo si les concierne o no (Mejías, 2021).

Las estaciones están conectadas por un único segmento de cable. A diferencia del anillo, el bus es pasivo, no se produce regeneración de las señales en cada nodo. Los nodos en una red de "bus" transmiten la información (López y Zamora, 2019).

Topología de anillo: Las estaciones están unidas unas con otras formando un círculo por medio de un cable común. Las señales circulan en un solo sentido alrededor del círculo, regenerándose en cada nodo. Cada nodo examina la información que es enviada a través del anillo (López y Zamora, 2019).

En esta red los dispositivos están conectados en un anillo. Los mensajes se transmiten de un ordenador a otro circulando en un bucle cerrado en una sola dirección. Puesto que cada ordenador trabaja de forma independiente (Beynon, 2018).

\section{MATERIALES Y METODOS}

El presente trabajo se ha considerado orientarlo por el método descriptivo con énfasis en la investigación aplicada, debido a que tiene como finalidad solucionar un problema, por lo que sus resultados se traducen en soluciones a manera de productos tecnológicos tangibles o intangibles (Zapatero Campos, 2010).

\section{RESULTADOS}

La implantación de un servidor de impresiones ayudará a la empresa a llevar una mejor gestión del servicio de las impresoras contando con complementos útiles; estableciendo conexiones hacia las máquinas de la red con mayor agilidad y rapidez. De tal manera, que al estructurar la red las impresiones pases por un servidor, el cual evitará el problema de impresiones erróneas, o impresiones basura, pues el usuario servidor tendrá la posibilidad de conceder la autorización para continuar con el 
proceso o caso contrario evitar la impresión de documentos no deseados y con órdenes equivocas.

\section{Misión de empresa}

Somos una organización privada que proporciona a estudiantes, profesionales, y público en general servicios tecnológicos tiene como misión el servir de apoyo y satisfacer las necesidades de los clientes con responsabilidad calidad y asesoramiento técnico apegándose a los planes estratégicos de desarrollo de la empresa.

\section{Visión de la empresa}

Consolidar a nuestra empresa como un organismo particular líder y pionero en los avances tecnológicos acordes a las necesidades de cambio que nos exige la globalización manteniendo altos niveles de calidad, solidez y eficacia en los diversos planes de acción tanto internos como externos orientados a dar servicio a la sociedad. Para mejorar la gestión del servicio de impresiones en el centro de cómputo Lumar system's se creará una red en árbol donde se utilizará dos switches de 8 puertos para conectar las diez máquinas y el server, los cuales estarán conectados a un router Dlink donde estarán conectadas las impresoras con cuenta el establecimiento, este router se conectará con el ADSL de CNT, quien es el proveedor de internet.

Para la red utilizaremos cable estructurado utp categoría 5, con terminales Rj45 ponchados con una configuración $568^{\mathrm{a}}$ la cual es una configuración para redes de tipo estándar y presta las seguridades y comunicación de datos necesarias para nuestra propuesta

El centro de Cómputo Lumar System's, cuenta con 10 estaciones de trabajo en el área, se requiere la conexión en red de dichos equipos para tener un acceso a los recursos que se encuentran separados como el servicio de impresiones.

Bajo este esquema se denomina Red de Área local, comúnmente conocida como LAN, por estar circunscrita a un área determinada, en este caso el centro de cómputo. 
Se presentan los elementos y su ubicación optima dentro de las instalaciones, para la puesta en marcha de dicha red.

\section{Topología}

Dentro de la topología de la red se considera la organización o distribución física de los equipos, cables y otros componentes de la red. La topología es el término estándar que utilizan la mayoría de los profesionales de las redes cuando se refieren al diseño básico de la red. La topología de una red afecta a sus capacidades. La selección de una topología tendrá impacto sobre: el tipo de equipamiento que necesita la red, las capacidades del equipo, el crecimiento de la red y las formas de gestionar la red.

Todos los diseños de redes parten de tres topologías básicas: bus, estrella, anillo; para más información de estas topologías referirse al Capítulo "Marco Teórico". En el desarrollo de la red del Centro de cómputo se sugiere la topología en estrella, justificando esta decisión por la razón: "que bajo este esquema el sistema solo colapsara en el supuesto que el punto central de la misma falle".

Teniendo, además las ventajas siguientes:

Si falla un equipo (o el cable que lo conecta al swich), el equipo afectado será el único que no podrá enviar o recibir datos de la red. El resto de la red continuara funcionando normalmente.

La modificación del esquema de la red y la incorporación de nuevos equipos es fácil.

Es posible un monitoreo y mantenimiento centralizado.

\section{Inventario}

La institución cuenta con equipo apto para la aplicación de este diseño.

Esto significa equipo adicional al centro de Cómputo.

Las características estándar de los mismos se describen a continuación:

\section{Hardware}


Servidor Proxy Pc Intel, procesador core TM i3 - 10100E a 3.20 Ghz con 8Gb en RAM 500 GB en disco duro sólido, y unidad de DVD-Writer. Tarjeta de red Fast Ethernet 10/100.

Estaciones de trabajo Pc Intel, procesador core TM i3 - 10100E a 3.20 Ghz con 8Gb en RAM 500 GB en disco duro sólido, y unidad de DVD-Writer. Tarjeta de red Fast Ethernet 10/100.

\section{Software}

Sistema Operativo Windows 10, Antivirus Trend Micro, Utilitarios Office 365

Nota: La descripción de Hardware y Software han sido tomados de las existencias físicas del centro de cómputo.

\section{Tamaño de las Instalaciones}

La distribución de las instalaciones está dividida en dos partes, denominadas zona de clientes y zona de mantenimiento de equipos. La zona de clientes, es la parte de acceso a las máquinas de alquiler donde se prestan los servicios de internet, consultas, transcripciones e impresiones a los diferentes clientes; se encuentran, además, el área administrativa, y el área para vender artículos de papelería y útiles Escolares. La zona de mantenimiento está compuesta por el área donde se brinda el servicio mantenimiento preventivo y correctivo de los equipos del centro de cómputo y de clientes que requieran de este servicio, zona donde se cuenta con tres puntos de conexión de internet. Las zonas han sido concebidas de tal manera por la estructura física del lugar, ya que hay unas gradas que llevan a la segunda planta la misma que separa ambas instalaciones. 


\section{CIENCIAMATRIA}

Revista Interdisciplinaria de Humanidades, Educación, Ciencia y Tecnología Año VII. Vol. VII. N¹. Edición Especial. 2021 Hecho el depósito de ley: pp201602FA4721

ISSN-L: 2542-3029; ISSN: 2610-802X

Universidad Nacional Experimental Francisco de Miranda (UNEFM). Santa Ana de Coro. Venezuela

Luis Orlando Albarracín-Zambrano; Juan Carlos Lozada-Calle; Jennifer Pamela Martínez-Matute

\section{Requerimientos de la Red}

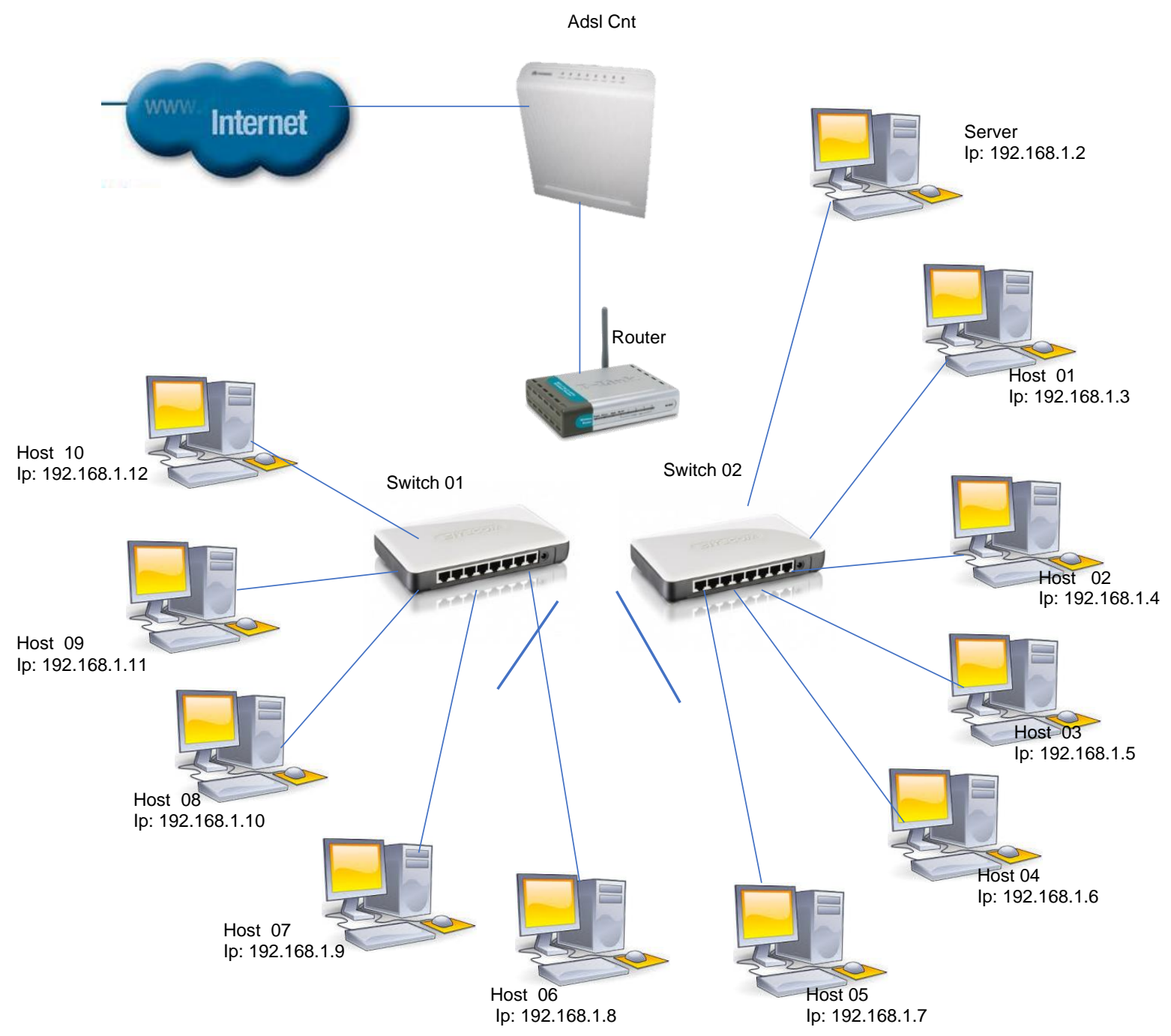


Figura 1: Distribución propuesta para la red del centro de cómputo Lumar System's Elaboración: Los autores.

\section{Selección del Cableado de Red}

Actualmente, la gran mayoría de las redes están conectadas por algún tipo de cableado, que actúan como medio de transmisión por donde pasan las señales entre los equipos. Hay disponibles una gran cantidad de tipos de cables para cubrir las necesidades y tamaños de las diferentes redes, desde las más pequeñas a las más grandes. Dentro de este punto se hará una selección de cable que se sugiere para la red. Se ha visto por la distribución de espacio en donde se colocaran las terminales, el cual es necesario utilizar el siguiente tipo de cable:

Cable de par trenzado sin apantallar (UTP) Categoría 5e.

Este cable ha sido seleccionado para comunicar la señal entre el router los swichs y las estaciones de trabajo dentro de la Zona de clientes y la Zona de mantenimiento. EI UTP, con la especificación 10baseT, es el tipo más conocido de cable de par trenzado y ha sido el cableado LAN más utilizado en los últimos años. El segmento máximo de longitud es de 100 metros. Los pares se trenzan para reducir la interferencia entre pares adyacentes. Admite frecuencias de hasta $100 \mathrm{Mhz}$.

Elementos Complementarios para una Conexión con UTP Cat5e

Para la correcta instalación del cable, se necesitan accesorios complementarios tales como:

Placas para Conectores: Estos se sitúan en la pared, permiten dos o más enganches para conectores de datos o de voz (telefónico).

Conectores RJ-45: El cable de par trenzado utiliza conectores RJ-45 para conectar a un equipo, estos son similares a los conectores telefónicos.

Patch Cord: Son cables de conexión de red. Su punta termina en un RJ-45 macho. Que se conectan en paneles de conexiones y placas de pared y alcanzan velocidades de datos de hasta 100 Mbps. 
Canaletas: Las canaletas son tubos metálicos o plásticos que conectados de forma correcta proporcionan al cable una segunda pantalla o protección. Estos deben ir fijos protegiendo el recorrido del cable de la red.

Swich: El swich es el componente hardware central de una topología de estrella, un conmutador que recibe/reespide paquetes de información. El swich solo opera en redes locales y nunca conecta dos redes, sino que segmentos de la misma red. Es pues más rápido y muy manejable. Permite administrar el ancho de banda entre los usuarios, posibilitando la creación de redes.

Tarjetas de Red: Las tarjetas de red, también conocidas NIC (Network Interfase Card, tarjetas de interfaz de red), actúan como la interfaz o conexión física entre el equipo y el cable de red. Las tarjetas están instaladas en una ranura de expansión en cada uno de los equipos.

Después de instalar la tarjeta de red, el cable de red se une al puerto de la tarjeta, para realizar a conexión física entre el equipo y el resto de la red.

La función de la tarjeta es:

Preparar los datos del equipo para el cable de red.

Enviar los datos a otro equipo.

Controlar el flujo de datos entre el equipo y el sistema de cableado.

Recibir los datos que llegan por el cable y convertirlos en bites para que puedan ser comprendidos por la unidad de procesamiento central del equipo (CPU).

La tarjeta de red que poseen los equipos a conectar a la red es: Fast Ethernet 10/100 Conocimientos Técnicos de los Usuarios: Los usuarios de esta red son los clientes que necesiten realizar algún tipo de actividad como consultas, redacción de documentos, o impresión de los mismos, así como también personal administrativo y de mantenimiento. Todos estos, están capacitados para hacer uso del equipo que le sea asignado.

Nivel de Tráfico de la Red: Se ha proyectado actualmente 10 puntos de red, teniendo en cuenta una expansión a futuro de 16 puntos de red.

Seguridad en la Red: Es necesario que exista un nivel de seguridad dentro de la red, los puntos más destacados son los siguientes: 
Seguridad de la Información: Para tener un nivel de seguridad en la información que se procesa en las diferentes estaciones de trabajo, es necesario tener en cuenta las siguientes recomendaciones:

a) Cada terminal de la red debe tener un usuario responsable de la misma.

b) Establecer permisos a los recursos que tengan acceso cada uno de los usuarios, ya sea de lectura, acceso total o dependiendo de la asignación.

Seguridad Física: Se deben tener algunas consideraciones para proteger la seguridad física de los elementos que componen la red.

a) Tener equipo de respaldo de energía eléctrica en los equipos que lo requieran.

b) Tener extintores en lugares estratégicos y asegurarse que estos se encuentren en buenas condiciones.

c) Capacitación a personal estratégico al buen uso de los extintores.

d) Seguir las indicaciones de las señales de evacuación a las zonas de seguridad en caso de desastre.

Seguridad en el Equipo: En un entorno de red debe asegurarse el buen funcionamiento de todo el equipo, siguiendo las siguientes directrices:

a) Solo usuarios autorizados deben tener acceso a programas y archivos confidenciales.

b) No permitir el uso del equipo por usuarios sin conocimiento del manejo de los equipos de cómputo.

c) Tener una rutina de supervisión física del uso de estaciones de trabajo y llevar una estadística de las revisiones.

d) Monitorear periódicamente la utilización de las estaciones de trabajo.

Medidas de Seguridad Adicionales: El administrador de la red puede incrementar el nivel de seguridad de una red de diversas formas.

Se ha proyectado que todas las terminales tengan un acceso dedicado a Internet, por lo que requiere una protección de un servidor Proxy. Se hace esta sugerencia por el hecho que la Institución obtendrá un equipo que se utilizará para tal fin.

Un servidor Proxy es un cortafuego (firewalls) que gestiona el tráfico de Internet que genera una red de área local (LAN). El servidor Proxy decide si es seguro permitir que 
un determinado mensaje pase a la red de la institución. Proporciona control de acceso a la red, filtrado y descarte de peticiones que el propietario no considera apropiadas, incluyendo peticiones de acceso sobre datos de propiedad.

Protección de los Virus: La proliferación de las LAN y el crecimiento de Internet han abierto muchas vías infección rápida de virus. Hoy en día, cualquier equipo en el mundo puede estar conectado a cualquier otro equipo como consecuencia, también se ha producido un aumento importante en el proceso de creación de virus. De hecho, algunos creadores de virus proporcionan software de fácil uso que contiene direcciones de cómo crear un virus.

Un virus puede causar mucho daño en el equipo. Por tal razón, una estrategia efectiva de antivirus constituye una parte esencial de la planificación de la red. Resulta esencial un buen antivirus. Dado que en este tema la prevención es la clave, se proponen las siguientes medidas:

a) Contraseñas con el objetivo de reducir la posibilidad de acceso no autorizado.

b) Desarrollar una lista que especifique el software a cargar.

c) Proteger todas las estaciones de trabajo con antivirus.

d) Mantener al día las actualizaciones del antivirus.

e) Asegurarse que todos los usuarios estén bien informados de los virus informáticos y de cómo evitar la activación de dichos virus.

\section{Router}

DIR-600 Wireless 150 Router

Características principales

Recepción Enhanced Wireless y Cobertura Basado en la tecnología 802.11n Draft.

Mejor Tecnología inalámbrica, hasta 4 veces más rápido que el estándar $802.11 \mathrm{~g}$.

Requiere Internet explorer 6 or firefox 2.0 o Superior.

Fácil de usar, características de seguridad inalámbrica con protección de firewall activo.

Requiere: Cable or Dsl modem. 
Requiere: Windows XP sP2 or Windows 2000 sP4 or mac os X (v10.4/v10.3) o sistema operativo linux-based.

\section{DISCUSIÓN}

Esta investigación permite presentar una solución desde el ámbito informático al control y configuración de un centro de cómputo y el manejo eficiente del proceso de impresiones lo cual permitirá una reactivación económica la cual se ha visto afectada por la pandemia del Covid 19, esta propuesta si bien está enfocado en el Centro de cómputo Lumar de System's, puede ser aplicado a las Pymes de cualquier sector o ciudad que tengan una actividad económica similar, o en un conjunto de oficinas que requieran el control de impresiones a través de un servidor.

La administración de recursos y organización estructurada de una red es necesaria para la optimización de recurso, control de seguridad de red, control de flujo de datos, manejo y administración de archivos, la distribución segmentada de internet con prioridades de usuarios y necesidades de consumo y conectividad, este proyecto se viabiliza por la importancia que hoy en día a tenido la tecnología en el desarrollo de una empresa ya sea privada o pública.

La aplicación del proyecto ayudara a la empresa "Centro de Computo Lumar System's" a llevar un mejor control en la gestión de impresiones y lograra reducir gastos en papelería, además por ser una empresa dedicada a prestar los servicios de internet, anillados, consultas, transcripciones, e impresiones, ayudara a que los usuarios por desconocimiento del proceso de impresión, cometan errores como, enviar múltiples ordenes de impresión ocasionando congestión en la cola de impresiones, impresiones de información no útil, representando para la empresa un mejoramiento en la gestión de administración, en el proceso de cobro de sus servicios y en la administra de los recursos que brinda la empresa. El administrador o el personal a cargo verificara y comprobara la orden de impresión y procederá a realizar el proceso, se evitará que usuarios inexpertos envíen impresiones no necesarias que por error sean enviadas y al momento de realizar la transacción de cobro se presenten inconvenientes. Los clientes se beneficiarán con la implementación del servidor de 
impresiones al reducir el pago por utilizar este servicio, evitando que por error envíen a la cola de impresiones documentos como demasiadas hojas sin información útil o "impresiones basura" de forma directa.

\section{CONCLUSIONES}

En los estudios realizados sobre las bases teórica en las que se basa la investigación se han considerado autores que desarrollan proyectos tecnológicos y configuraciones de rede de comunicación y flujo de datos, los cuales constituyen en la creación de una base de conocimientos para la solución de problemáticas informáticas

La metodología de la investigación permitió identificar la problemática y segregar los componentes para desde un análisis tecnológico comprender las mejores posibilidades para solucionar una problemática general desde un punto de vista particular del centro de cómputo Lumar System's.

La propuesta planteada se convierte en un modelo aplicable para la gestión de impresión dentro de una red, el cual puede ser utilizado en las empresas para economizar recursos y mejorar sus servicios hacia los usuarios o clientes de las Pymes.

FINANCIAMIENTO: No monetario.

\section{AGRADECIMIENTO}

Agradecemos a los colaboradores de esta investigación, a los gerentes y trabajadores de la empresa “Centro de Computo Lumar System's” del Cantón la Maná.

\section{REFERENCIAS CONSULTADAS}

Arias, V; Herrera, J; Gómez, J; Jaramillo, P; Tilero, R. (2019). Implementación de servicios de infraestructura TI corporativa con Zentyal Server 5.Ariculo en línea: https://repository. Unad.edu.co.

Bordignon, F. e Iglesia, A. (2020). Introducción al Pensamiento Computacional. La edición para educar. UNIPE. Editorial Universitaria. Buenos Aires. Argentina. 
Beynon, P. (2018). Sistema de Información. Introducción a la Informática en las Organizaciones. Edición 1. Editorial REVERTE. Barcelona, Bogotá, Buenos Aires, Caracas.

Domínguez, S. E. (2018). Circuitos electrónicos auxiliates de vehículo. España: EDITEX.

Dormido, S; Sánchez, J y Morilla, F. (2000). Laboratorios virtuales remotos para la práctica a distancia de la automática. XXI Jornadas de Automática, Conferencia plenaria, Sevilla. España.

López, J y Zamora, D. (2019). Diseño de una red lan para la empresa Multiweb. Revista Repositorio Institucional. Universidad de Colombia.

Mejías, M; Arias, A; Castillo, A; Roa, A; Bidó, E; García; J; Hernández, D. (2021). Protocolos y topologías utilizadas en los sistemas de comunicación de las microrredes eléctricas. Revista Ciencia, Ingenierías y Aplicaciones, Vol. 4(1), 8195.

Moliner, F. (2005). Grupo A y B de Informática Bloque Específico, Vol. 2- ValenciaEspaña. Madr S.L.

Sánchez, Y; Botaños, C. (2021). Diseño y simulación de una red de comunicaciones de conexión punto a multipunto de topología anillo para conectar a tres sedes de la institución educativa Departamental Sagrado Corazón de Jesús de PivijayMagdalena. Revista Repositorio Institucional. Universidad de Colombia

Zapatero Campos, J. A. (2010). Fundamentos de investigación para estudiantes de ingeniería (Tercer Escalón, Ed.).

\footnotetext{
(C2021 por los autores. Este artículo es de acceso abierto y distribuido según los términos y condiciones de la licencia Creative Commons Atribución-NoComercial-Compartirlgual 4.0 Internacional (CC BY-NC-SA 4.0) (https://creativecommons.org/licenses/by-nc-sa/4.0/).
} 